\title{
Quantized, piecewise linear filter network
}

\section{Sørensen, John Aasted}

\section{Published in:}

Proceedings of the IEEE-SP Workshop Neural Networks for Signal Processing

Link to article, DOI:

10.1109/NNSP.1993.471841

Publication date:

1993

\section{Document Version}

Publisher's PDF, also known as Version of record

Link back to DTU Orbit

Citation (APA):

Sørensen, J. A. (1993). Quantized, piecewise linear filter network. In Proceedings of the IEEE-SP Workshop Neural Networks for Signal Processing (pp. 470-474). IEEE. https://doi.org/10.1109/NNSP.1993.471841

\section{General rights}

Copyright and moral rights for the publications made accessible in the public portal are retained by the authors and/or other copyright owners and it is a condition of accessing publications that users recognise and abide by the legal requirements associated with these rights.

- Users may download and print one copy of any publication from the public portal for the purpose of private study or research.

- You may not further distribute the material or use it for any profit-making activity or commercial gain

- You may freely distribute the URL identifying the publication in the public portal

If you believe that this document breaches copyright please contact us providing details, and we will remove access to the work immediately and investigate your claim 


\title{
Quantized, Piecewise Linear Filter Network.
}

\author{
John Aasted Sørensen, Electronics Institute, Build 349 \\ Technical University of Denmark, Lyngby \\ DK-2800 Denmark \\ email: jaas@dthei.ei.dth.dk; fax: +4542880117
}

\begin{abstract}
A quantization based piecewise linear filter network is defined. For stationary signals this filter network is a generalization of the classical Wiener filter, with an input signal $x_{n}$ and a desired response $d_{n}$, $n=1,2, \ldots$. The object of the piecewise linear filter network, is the approximation of a possible nonlinear function $G(x)$ between $x_{n}$ and $d_{n}$. A method for the training of this network based on local approximation in the input space is devised. The training is carried out by repeatedly alternating between vector quantization of the training set into quantization classes and equalization of the quantization classes linear filter mean square training errors. The equalization of the mean square training errors is carried out by adapting the boundaries between neighbor quantization classes such that the differences in mean square training errors are reduced.
\end{abstract}

\section{Introduction}

A quantization based, piecewise linear filter network is defined. For stationary signals this filter network is a generalization of the classical Wiener filter [1], with an input signal $x_{n}$ and a desired response $d_{n}, n=1,2, \ldots$ The object of the piecewise linear filter network [2] is the approximation of a possible nonlinear function $G(x)$ between $x_{n}$ and $d_{n}$, as shown in Fig. 1. The training of the filter network is carried out by repeatedly alternating between vector quantization of the training set $\left\{x_{n}, d_{n}, n=1, \ldots, N\right\}$ into quantization classes $\mathcal{Q}_{i}$ and equalization of the quantization classes linear filters mean square training errors $J_{i}$, by adapting the boundaries between neighbor quantization classes, such that the differences in mean square errors is reduced. 
The optimization criterium used in the training of the filter network is

$$
\begin{gathered}
J=\sum_{i} J_{i}+\frac{1}{2} \sum_{(i, j)}\left|J_{i}-J_{j}\right| \\
i: \text { quantization classes } \\
(i, j): \text { neighbor quantization classes }
\end{gathered}
$$

The first sum in $J$ reduces the total training error of the linear filters $\mathbf{w}_{i}$, associated the quantization classes and the second sum equalize the training errors by controlling the boundaries between neighbor quantization classes. Piecewise linear filters are given in [3], [4] and [5]. In [3] the local linear filters are constructed for each partition resulting from a vector quantization. In [4] a piecewise linear filter is devised, based on the "canonical piecewiselinear (PWL) structure" and [3] is based on a scalar quantization of the filter outputs, where the filters are organized in a tree structure.

\section{The Constituent Elements of the Training Al- gorithm}

$\left\{x_{n}, d_{n}, n=1, \ldots, N\right\}$ : Training set.

$M$ : Dimension of input space.

$\mathbf{x}_{n}^{\prime}=\left(x_{n}, x_{n-1}, \ldots, x_{n-M+1}\right)$ where ' denotes transposed.

$\mathbf{X}=\left[\mathbf{x}_{n}^{\prime}\right] \in \mathbf{R}^{(N-M+1) \times M}$

$\mathbf{x} d_{n}^{\prime}=\left[\mathbf{x}_{n}^{\prime}, d_{n}\right]$

$\mathbf{X} \boldsymbol{d}=\left[\mathbf{x} d_{n}^{\prime}\right] \in \mathbf{R}^{(N-M+1) \times(M+1)}$

$\operatorname{dist}\left(\mathbf{x} d_{m}, \mathbf{x} d_{n}\right)$ : Distance between $\mathbf{x} d_{m}$ and $\mathbf{x} d_{n}$.

$s$ : The number of quantization iterations.

$k$ : The number of equalization iterations at a given number of quantizations.

$Q_{s}:$ The number of quantization classes after $s$ quantization iterations.

$\mathcal{Q}_{i}=\left\{\mathbf{x} d_{i j} \in\right.$ quantization class no. $\left.i\right\}, j=1, \ldots, q_{i}, i=1, \ldots, Q_{s}$

$q_{i}$ : The number of training elements in quantization class no. $i$.

$\mathbf{J}^{k}=\left[J_{i}^{k}\right], i=1, \ldots, Q_{s}:$ Mean square error of the linear filter in quantization class no. $i$ at the equalization iteration no. $k$

$\mathbf{W}^{k}=\left[\mathbf{w}_{j}^{k}\right] \in \mathbf{R}^{(M+1) \times Q}$ : : linear filters of the quantization classes.

$\mathbf{C}^{k}=\left[\mathbf{c}_{j}^{k}\right] \in \mathbf{R}^{(M+1) \times Q}$ : : Mean values for quantization classes.

$\mathbf{Q}_{k}=\left[\mathbf{q}_{j}^{k}\right] \in\{0,1\}^{(N-M+1) \times Q_{s}}:$ Partition matrix for $\mathbf{X} d$.

$\mathbf{q}_{j}^{k}=\left[q_{i j}^{k}\right]$ where $q_{i j}^{k}=1$ if $\mathbf{x} d_{i} \in$ quantization class no. $j$. Otherwise $q_{i j}^{k}=0$.

$\mathbf{P}^{k}=\left[p_{i j}^{k}\right] \in\{0,1\}^{Q_{s} \times Q_{s}}:$ Neighbor matrix for quantization classes.

$p_{i j}^{k}=1$ if the quantization classes $i$ and $j$ are neighbors. Otherwise $p_{i j}^{k}=0$. 


\section{Training Algorithm Overview.}

The training algorithm shown in the next section, is based on two nested operations carried out iteratively. The first operation is vector quantization of the training set, represented by the matrix $\mathbf{X} d$. The result of this is the mean values of quantization classes $\mathbf{C}^{0}$, the training set partition matrix $\mathbf{Q}^{0}$, the neighbor matrix $\mathbf{P}^{0}$ of the quantization classes and the mean square training errors $\mathbf{J}^{0}$ of the quantization classes.

In the second operation, denoted equalization, the quantization classes are adapted through the operation adapt quantization classes according to the current training errors of the classes and their neighbor classes training errors. The two adaptation rules considered here are:

Rule 1: Adapt the current quantization class in the direction of the largest training error gradient of the neighbors to the current quantization class.

$$
\mathbf{c}_{i}^{k}=\mathbf{c}_{i}^{k-1}+\mu_{i j} \max _{j}\left\{\frac{h\left(J_{j}^{k-1}-J_{i}^{k-1}\right)}{J_{j}^{k-1}} p_{i j}^{k-1}\right\}\left(\mathbf{c}_{j}^{k-1}-\mathbf{c}_{i}^{k-1}\right)
$$

where: $i, j=1, \ldots, Q_{s}, h(x)=0.5(|x|+x), p_{i j}^{k-1}:$ neighbor matrix element and $\mu_{i j}$ is a constant.

Rule 2: Adapt the current quantization class in the direction of the mean training error gradient to the neighbors of the current quantization class.

$$
\begin{gathered}
\mathbf{c}_{i}^{k}=\mathbf{c}_{i}^{k-1}+\frac{1}{\eta_{i}} \sum_{j=1}^{Q s} \mu_{i j} \frac{J_{j}^{k-1}-J_{i}^{k-1}}{J_{j}^{k-1}} p_{i j}\left(\mathbf{c}_{j}^{k-1}-\mathbf{c}_{i}^{k-1}\right) \\
i, j=1, \ldots, Q_{s}
\end{gathered}
$$

$\eta_{i}$ is the number of neighbor quantization classes of class $i$.

Both rules leads to the transformation:

$$
\mathbf{C}^{k}=\mathbf{C}^{k-1} \Gamma^{k-1} \text { where } \Gamma^{k-1}=\left[\gamma_{i j}\right] \in \mathbf{R}^{Q \times Q} .
$$

Rule 1 leads to these properties of $\boldsymbol{\Gamma}$ :

- $0 \leq \gamma_{i j} \leq 1$

- $\gamma_{i j} \gamma_{j i}=0$.

- If $\gamma_{i i}=1$ then the quantization class no. $i$ has a local (global) maximum in the training error, compared to its neighbors. At least one $\gamma_{i i}$ will be equal to 1 .

- $\Gamma$ is a column stochastic matrix. (Each column sums to 1 ). 
Rule 2 leads to these properties of $\boldsymbol{\Gamma}$ :

- $\gamma_{i j}<1$

- If $\operatorname{sign}\left(\gamma_{i j}\right)=-1$ for $i=1, \ldots, Q_{s}, i \neq j$ then the quantization class $j$ is a local (global) maximum.

- If $\operatorname{sign}\left(\gamma_{i j}\right)=1$ for $i=1, \ldots, Q_{s}, i \neq j$ then the quantization class $j$ is a local (global) minimum.

- $\Gamma$ is a column stochastic matrix.

The adaptation is followed by a minimum distance classification of the training set using $\mathbf{C}^{k}$ and finally determination of the local filter matrix $\mathbf{W}_{k}$.

The results of the training algorithm are the quantization class means $\mathbf{C}^{k}$ defining the quantization classes and the local linear filters $\mathbf{W}_{k}$.

\section{The Training Algorithm.}

Initialize:

$s=0:$ The number of quantization iterations.

$k=0:$ The number of equalization iterations.

$J^{-1}=\infty, J^{0}=0$.

$\boldsymbol{\Lambda}^{0}$ : Identity partition for the training set.

$\boldsymbol{\Theta}^{0}$ : Mean value of the training set.

while $\left|J^{s-1}-J^{s}\right| / J^{s-1}>$ Quantize $_{\text {thres }}$

$s=s+1$

$\left(\mathbf{C}^{0}, \mathbf{Q}^{0}, \mathbf{P}^{0}, \mathbf{J}^{0}\right)=$ vector quantization $\left(\mathbf{\Lambda}^{k}, \Theta^{k}, \mathbf{X} d\right)$

$k=0$

while $\max _{(i, j)}\left|J_{j}^{k}-J_{i}^{k}\right| p_{i j}^{k} / J_{j}^{k}>$ Equalize $_{\text {thres. }}$

$k=k+1$

$\left(\mathbf{C}^{k}\right)=$ adapt quantization classes $\left(\mathbf{C}^{k-1}, \mathbf{Q}^{k-1}, \mathbf{J}^{k-1}\right)$

$\left(\mathbf{Q}^{k}, \mathbf{P}^{k}\right)=$ classify training $\operatorname{set}\left(\mathbf{X} d, \mathbf{C}^{k}\right)$

$\left(\mathbf{W}^{k}, \mathbf{J}^{k}\right)=$ quantization class filters $\left(\mathbf{Q}^{k}, \mathbf{X} d\right)$

$J^{k}=\sum_{i=1}^{Q,} J_{i}^{k}+0.5 \sum_{i=1}^{Q,} \sum_{j=1}^{Q,}\left|J_{i}^{k}-J_{j}^{k}\right| p_{i j}^{k}$

endwhile equalization

$J^{s}=J^{k}$

$\boldsymbol{\Theta}^{k}=\mathbf{C}^{k}$

$\mathbf{\Lambda}^{k}=\mathbf{Q}^{k}$

endwhile quantization

Result:

$\mathbf{W}_{s}^{k}$ : Quantization class filters.

$\mathbf{C}_{k}^{s}$ : Quantization class means. 


\section{A Training Example}

A training set is defined as follows:

$x_{n}$ : White, Gaussian noise, $\sigma_{x}^{2}=1$.

Prefiltering: $y_{n}=0.5 y_{n-1}+x_{n}$.

Scalar, 2-slope nonlinearity: $d_{n}=f\left(y_{n}\right)$ where:

$$
f(x)=\left\{\begin{array}{lcl}
-f(-x) & \text { for } & 0 \leq x \leq 0.5 \\
x & - & 0.5 \leq x
\end{array}\right.
$$

A training set with $N=200$, wdim $=2, \mu_{i j}=1.0$ leads to the following ratio between the mean square training error of the linear system and a quantized system with 4 quantization classes: 1.6 .

\section{References}

1. S. Haykin, "Adaptive Filter Theory", 2. Ed, Prentice-Hall, 1991.

2. J.Aa. Sørensen, "A Family of Quantization Based Piecewise Linear Filter Networks". IEEE ICASSP-92, March 23-26., San Francisco, USA.

3. Todd K. Leen, Nandakishore Kambhatla, "Fast Non-Linear Dimension Reduction." Neural Networks for Computing Conference.

Snowbird, Utah, USA, April 13-16. 1993.

4. Ji-Nan Lin, Rolf Unbehauen, "Adaptive Nonlinear Digital Filter with Canonical Piecewise-Linear Structure".

IEEE Trans. CAS-37, March 1990.

5. Saul B. Gelfand, C.S. Ravishankar, Edward J. Delp, "A Tree-Structured Piecewise Linear Adaptive Filter". IEEE ICASSP-91 pp.2141-2144.

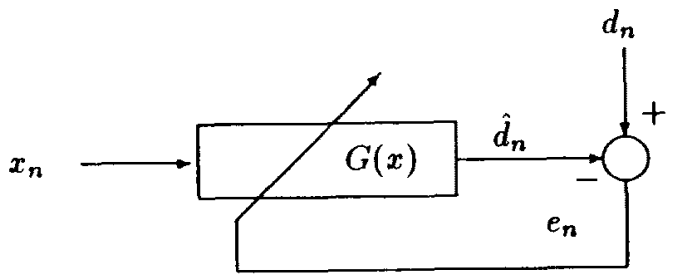

Figure 1: Adaptive filter modelling a nonlinear function $G(x)$ 